\title{
Tecnologías de la información en la formación de evaluadores del Programa Hospital Seguro en México
}

\author{
Information technologies in the training of evaluators of the Safe Hospital Program in \\ Mexico
}

\section{Felipe Cruz-Vega ${ }^{1}$, Juan C. Sánchez-Echeverría ${ }^{1 *}$, Hermes M. Cortes-Meza ${ }^{1}$, Jorge Loria-Castellanos ${ }^{1}$ y Erandy Montes de Oca-García ${ }^{2}$}

${ }^{1}$ División de Proyectos Especiales en Salud, Dirección de Prestaciones Médicas; ${ }^{2}$ Hospital General 197. Instituto Mexicano del Seguro Social (IMSS), Ciudad de México, México

\section{Resumen}

Objetivo: Describir el proceso para la formación de evaluadores del Programa Hospital Seguro centrado en el uso de las tecnologías de la información. Método: Estudio observacional descriptivo en el que se analiza la participación dentro del nuevo curso de evaluadores del Programa Hospital Seguro. Resultados: Se inscribieron 1323 participantes, de los cuales aprobaron 298 (18\%); la calificación media fue de 8.85. Conclusiones: La plataforma educativa tipo Moodle fue de utilidad para la capacitación del Programa Hospital Seguro. Se tendrían que mejorar los criterios de participación de los alumnos a fin de incrementar la eficiencia terminal.

PALABRAS CLAVE: Educación a distancia. Desastres. Programa Hospital Seguro.

\begin{abstract}
Objective: To describe the process for the training of evaluators of the safe hospital program in the use of ICT. Method: Descriptive observational study in which the participation in the new course of appraisers of the safe hospital program is analyzed. Results: 1323 participants registered of which 298 (18\%) passed; the average grade was 8.85. Conclusions: The Moodle type educational platform was useful for the training of the Safe Hospital Program. The criteria for student participation would have to be improved in order to increase terminal efficiency.
\end{abstract}

KEY WORDS: Distance education. Disasters. Safe Hospital Program.

\footnotetext{
Correspondencia:

*Juan Carlos Sánchez-Echeverría

Paseo de la Reforma, 476

Col. Juárez, Del. Cuauhtemoc

C.P. 06600, Ciudad de México, México

E-mail: juancarlosmarx@gmail.com
}

Fecha de recepción: 01-03-2018

Fecha de aceptación: 06-04-2018

DOI: 10.24875/CIRU.M18000036
Cir Cir. 2018;86:237-243

Contents available at PubMed www.cirugiaycirujanos.com 


\section{Introducción}

El eje central de un sistema de salud que se precia de proteger la vida es la prevención. Es también el reto que enfrentan los sistemas de gestión de desastres que procuran reducir el riesgo hasta niveles aceptables y así contribuir a un desarrollo sustentable de las poblaciones. Una respuesta no efectiva ante un desastre genera altos costos, en muchos casos incalculables, lo que se traduce en un empobrecimiento de la población y un estancamiento del desarrollo de los países y las regiones. La mitigación de los efectos producidos por los desastres mediante la aplicación de acciones preventivas es altamente rentable ${ }^{1}$.

Latinoamérica es la segunda región más afectada por desastres naturales. El $67 \%$ de sus establecimientos de salud están ubicados en zonas de riesgo. De 2004 a 2014, el 61\% de los hospitales han sido afectados por sismos, el $17 \%$ por huracanes, el $14 \%$ por inundaciones y el $8 \%$ por emergencias sanitarias (epidemias, contaminación interna y otras). Cerca de 24 millones de personas quedaron sin atención sanitaria por la afectación en los servicios de salud $(200,000 \text { personas por unidad })^{2}$.

En México se tiene como punto de inflexión la experiencia derivada del sismo de 1985. Las afectaciones a la infraestructura hospitalaria, sobre todo en la Ciudad de México, derivaron en la pérdida de capacidad de respuesta en atención médica en unidades de alta complejidad, pues se perdieron más de 5000 camas hospitalarias en menos de 70 segundos. Además del elevado número de víctimas que perecieron en las estructuras colapsadas de los hospitales, la mortalidad se vio incrementada por el colapso funcional de las unidades que quedaron en pie. El factor determinante fue un sector salud sin preparación en respuesta frente a desastres ${ }^{3}$.

A partir de las acciones de reconstrucción se desarrollaron iniciativas dirigidas a mejorar la resiliencia, considerada como la capacidad que tiene un sistema, una comunidad o una sociedad expuestos a una amenaza para resistir, absorber, adaptarse, transformarse y recuperarse de sus efectos de manera oportuna y eficiente, en particular mediante la preservación y la restauración de sus estructuras y funciones básicas por conducto de la gestión de riesgos ${ }^{4}$.

Bajo este precepto, la Organización Panamericana de la Salud (OPS) y la Organización Mundial de la Salud (OPS/OMS) desarrollaron la iniciativa Hospitales Seguros Frente a Desastres.
En 1997, el Instituto Mexicano del Seguro Social inició actividades con la OPS/OMS para encontrar un método confiable para implementar el diagnóstico y la seguridad en hospitales, entre las que pueden mencionarse $\mathrm{e}^{5,6}$ :

- 2004: en su resolución CD45.R8 instó a los Estados miembros a que adoptasen el lema «Hospitales Seguros Frente a Desastres» como una política nacional de reducción de riesgos.

- 2005: se aprobó el marco de acción de Hyogo para 2005-2015, en el que se hace referencia al "aumento de la resiliencia de las naciones y las comunidades frente a los desastres».

- 2006: México establece el Comité Nacional de Evaluación, Diagnóstico y Certificación del Programa Hospital Seguro.

- 2007: se desarrolla Hospitales Seguros, iniciativa regional sobre establecimientos sanitarios capaces de resistir los efectos de los desastres.

- 2008: se elabora un plan de acción regional de Hospitales Seguros aprobado en resolución CD50.R15 de 2010, dentro de su plan estratégico 2008-2012.

- Se establece como meta para 2015 la construcción de hospitales nuevos con un nivel de seguridad que garantice su capacidad de funcionamiento en situaciones de desastre.

- Durante la III Conferencia Mundial para la Reducción de Riesgos de Desastres (2015-2030) se adoptó el Marco de Sendai, instrumento sucesor del Marco de Acción de Hyogo, "Aumento de la resiliencia de las naciones y las comunidades ante los desastres".

El Programa Hospital Seguro es una iniciativa de la OPS/OMS y tiene un carácter netamente preventivo. Se fundamenta en una herramienta que es el Índice de Seguridad Hospitalaria, el cual se obtiene a partir de una evaluación visual y objetiva por un grupo selecto de profesionales relacionados con el área de la salud.

Para llevar a cabo esta evaluación se utiliza la lista de verificación propuesta por la OPS/OMS, la cual describe los criterios para que cada punto sea calificado como bajo, medio o alto. A partir de esta valoración se aplica un modelo matemático para obtener el índice de seguridad hospitalaria. Este protocolo de evaluación está sistematizado en cuatro rubros: ubicación geográfica, seguridad estructural, seguridad no estructural y seguridad funcional; lo que permite una valoración integral de la instalación, así como de la organización. Los hospitales son clasificados 
mediante una cédula, según su capacidad instalada para responder frente a emergencias o desastres.

En agosto de 2007 se dio inicio al curso «Evaluadores del Programa Hospital Seguro", el cual se impartía vía Internet y estaba integrado por 33 temas contenidos en 60 horas lectivas. La dinámica consistía exclusivamente en la revisión de contenidos a través de videos, para al final de los mismos realizar una evaluación. En esta modalidad se realizaron 21 periodos de capacitación, con un registro de 7474 participantes, de los cuales aprobaron 1792 (23.97\%),8.

Esta capacitación de los evaluadores reviste una gran complejidad, tanto administrativa como logística. Se identificó, a partir de la variabilidad en la aplicación de la lista de verificación, que el proceso de enseñanza-aprendizaje no estaba centrado en el alumno (futuro evaluador). El proceso, en muchos casos, era limitativo al aprendizaje por imitación, principalmente porque el evaluador no podía desarrollar la función de retroalimentación, debido a que el método de enseñanza se basaba en un modelo vertical, parecido al modelo clásico. Del mismo modo, se observó que estaba ausente la posibilidad de compartir experiencias con otros evaluadores de otras instituciones, debido a que no existía el foro designado para ello.

A 8 años de su implementación, y considerando tanto la actualización de temas y contenidos como el crecimiento y la influencia del programa, consideramos necesario desarrollar un proceso de mejora con miras a satisfacer las necesidades educativas y administrativas en todos sus procesos. Para ello, a partir de un análisis de procesos realizado de abril a junio de 2016, se consideró la pertinencia del uso de las herramientas tecnológicas disponibles. A continuación describimos la experiencia de esta implementación.

\section{Método}

El curso se desarrolló en una plataforma virtual (http://cvoed.imss.gob.mx/HospitalSeguro/login/index.php) tipo Moodle (Modular Object-Oriented Dynamic Learning Environment) que contiene un ambiente virtual para la capacitación, la gestión y la administración de recursos y datos.

Entre las bondades que nos motivaron a emplear una plataforma tipo Moodle se encuentran: una organización modular, multiherramienta, que contribuye al desarrollo y la actualización tecnológica del programa; permitir el acceso a contenidos desde cualquier parte del mundo; facilitar el trabajo colaborativo con otras plataformas (p. ej., OPS); posibilidad de visualizarse desde cualquier dispositivo electrónico, además de integrar una red de recursos, información e instrumentos para los evaluadores en campo; opción de ser expandible en recursos y actividades, de acuerdo con las necesidades que se requieran, además de ser intuitiva, amigable y de acceso controlado y seguro?.

El curso constó de 12 módulos: 1) Generalidades; 2) Sistema Nacional de Protección Civil; 3) Sistema Nacional de Salud; 4) Gestión integral de riesgos en México; 5) Metodología para la evaluación hospitalaria; 6) Índice de Seguridad Hospitalaria; 7) Identificación geoespacial de peligros y riesgos. Atlas Nacional de Riesgos; 8) Seguridad estructural; 9) Seguridad en elementos no estructurales; 10) Capacidad funcional; 11) Hospital resiliente; y 12) Evacuación de hospitales con énfasis en áreas críticas. En total fueron 60 horas lectivas.

Cada tema se desarrollaba a través de libros electrónicos de contenidos desarrollados por expertos en la materia, y contaban con la validación conceptual y de contenidos por expertos en desastres, urgencias y educación.

Previamente a la implementación del curso se llevó a cabo la prueba piloto en 126 evaluadores expertos.

La estrategia de instrucción consideraba:

- Revisión y análisis de las instrucciones en cada módulo.

- Lectura y comprensión de los contenidos en el libro electrónico.

- Actividades de retroalimentación.

- Aprobar las evaluaciones modulares.

- Aprobar la evaluación final.

Un punto clave lo constituyó el tutor en línea, el cual estaba disponible las 24 horas del día a través de chat y de foros, para orientar y para resolver dudas, tanto de los temas como de cuestiones de la plataforma.

Los alumnos participantes fueron propuestos por cada una de las instituciones involucradas en el programa, y consideró a personal médico, paramédico, técnico y administrativo, todos involucrados directamente en la planeación y la respuesta ante situaciones de emergencia o desastres.

\section{Resultados}

Se registraron 1323 participantes de las diferentes instituciones del país; de ellos, 884 (55\%) tuvieron actividad y 298 aprobaron (18\%); 439 de los inscritos $(27 \%)$ no ingresaron a la plataforma en ninguna 
ocasión. Aprobaron alumnos de 23 de los 32 Estados del país (Tabla 1). En los 298 alumnos aprobados, la calificación fluctuó entre 8.00 y 9.86, con una media de 8.85. De los 12 módulos, en el que hubo un mayor porcentaje de no probados fue en el de Seguridad Estructural (módulo 8). La duración total del curso fue de 8 semanas. El módulo 8 mantuvo en promedio 9 días de actividad. Las principales participaciones en los foros estuvieron dirigidas a los módulos 4 y 8 .

\section{Discusión}

El objetivo es fortalecer la preparación para la respuesta en caso de emergencias y desastres, y lograr que la respuesta de las instituciones sea ordenada, sistemática y eficiente. Esto en virtud de que existen estudios que documentan, en varios países, que las unidades hospitalarias suelen no estar preparadas para responder ante un incidente de saldo masivo de víctimas o desastres, por la falta de conocimiento, escasez de planes de desastres y limitación de los recursos.

La versión de 2017 del curso para evaluadores del Programa Hospital Seguro se desarrolló basándose en un entorno de educación virtual tipo Moodle, proporcionado gratuitamente como programa de código abierto bajo Licencia Pública General GNU. Cualquier persona puede adaptar, extender o modificar Moodle, tanto para proyectos comerciales como no comerciales, sin pago de cuotas por licenciamiento, y beneficiarse del costo/beneficio, la flexibilidad y otras ventajas de usar Moodle. En la actualidad, globalmente Moodle cuenta con más de 79 millones de usuarios ${ }^{7}$, entre académicos y empresariales, lo que la convierte en la plataforma de aprendizaje más utilizada en el mundo. Este fue uno de los criterios de elegibilidad, además de tratarse de software libre y de ser una plataforma poderosa, con múltiples herramientas para la educación, segura y con actualizaciones frecuentes ${ }^{9}$.

Llama la atención que más de la cuarta parte de los inscritos no accedió al curso; en esto tendremos que considerar si se trató de una falta de coordinación con las instituciones que los propusieron o simplemente por falta de interés.

Entre los objetivos de los ambientes de capacitación está el brindar soporte técnico y tecnológico al curso virtual para la formación de evaluadores v2.02017, así como proporcionar capacitación continua y actualización en temas relacionados a los evaluadores del programa, además de estimular un ambiente de colaboración para generar trabajos y publicaciones en emergencias y desastres.

El Grupo Técnico Asesor del Comité Nacional de Evaluación, Diagnóstico y Certificación del Programa Hospital Seguro propuso que el nuevo curso para la formación de evaluadores del Programa Hospital Seguro migrara a la plataforma Moodle, en gran parte motivados por las muchas cualidades que posee y que fortalecerían el proceso de educación a distancia, entre las que destacan:

- Interfaz moderna, fácil de usar, diseñada para ser responsiva y accesible. La interfaz de Moodle es fácil de navegar, tanto en computadoras de escritorio como en dispositivos móviles.

- Cuenta con un tablero personalizable para mostrar cursos, mensajes y tareas actuales.

- Actividades y herramientas colaborativas. Se dispone de herramientas para trabajar y aprender juntos en foros, wikis, glosarios, actividades de base de datos y mucho más.

- Calendario todo en uno. La herramienta del calendario de Moodle permite mantener al día el calendario académico o el de la compañía, fechas de entrega dentro del curso, reuniones grupales y otros eventos personales.

- Gestión conveniente de archivos. Permite arrastrar y colocar archivos desde servicios de almacenamiento en la nube, incluyendo MS OneDrive, Dropbox y Google Drive.

- Editor de texto simple e intuitivo. Puede darse formato convenientemente al texto y añadir multimedia e imágenes con un editor que funciona con todos los navegadores de Internet y en todos los dispositivos.

- Notificaciones. Cuando se habilitan, los usuarios pueden recibir alertas automáticas acerca de nuevas tareas y fechas para entregarlas, así como realizar publicaciones en foros y mandarse mensajes privados entre ellos.

- Monitoreo del progreso. Los educadores y los educandos pueden monitorear el progreso y el grado de finalización con un conjunto de opciones para el monitoreo de actividades individuales o recursos, y también del curso.

- Poderosas herramientas administrativas para la gestión de cursos, usuarios y contenidos.

El nuevo curso para formación de evaluadores del Programa Hospital Seguro fue rediseñado totalmente en sus contenidos para contextualizarlo en el marco de la gestión integral de riesgos ante amenazas múltiples, atendiendo a las recomendaciones del Marco 

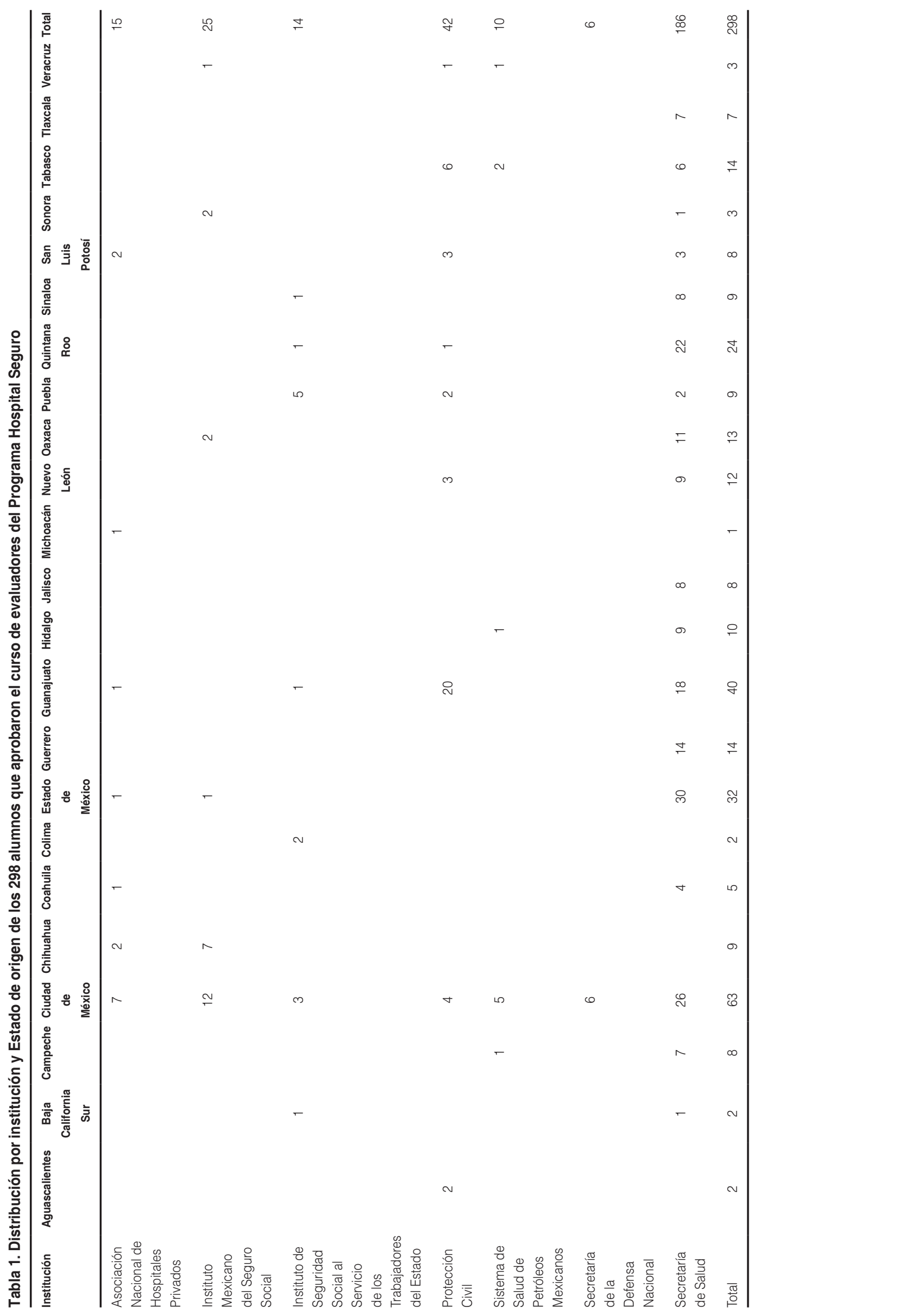
de Sendai en el que se estableció que es urgente y fundamental prever el riesgo de desastres, planificar medidas y reducirlo para proteger de manera más eficaz a las personas, las comunidades y los países, sus medios de subsistencia, su salud, su patrimonio cultural, sus activos socioeconómicos y sus ecosistemas, reforzando así su resiliencia.

La actualización del curso 2017 intenta dar respuesta a la premisa de que es urgente fortalecer y mantener un enfoque preventivo del riesgo de desastres más amplio y más centrado en las personas, en el que las prácticas de reducción del riesgo de desastres contemplen amenazas múltiples y sean multisectoriales, inclusivas y accesibles para resultar eficientes y eficaces, por lo que los sectores público y privado, y las organizaciones de la sociedad civil, así como la comunidad académica y las instituciones científicas y de investigación, deben colaborar más estrechamente y crear oportunidades de colaboración, y las empresas tienen que integrar el riesgo de desastres en sus prácticas de gestión.

En el nuevo curso se incluyó el abordaje del tema "Gestión integral de riesgos en México", cuyo objetivo es identificar los aspectos básicos de riesgo, así como su gestión integral, adentrándose en las etapas que conforman dicha gestión e identificando los instrumentos existentes.

En este módulo se expone cómo la reducción del riesgo de desastres es una inversión rentable en la prevención de pérdidas futuras; además, una gestión eficaz del riesgo de desastres contribuye al desarrollo sostenible.

Otro tema que no se había contemplado en las versiones anteriores del curso es «Hospital resiliente", que ahora se incluye con el objetivo de conocer el nuevo concepto de hospitales frente a un contexto de multiamenaza, desde un enfoque dirigido a la gestión y la reducción del riesgo de desastre, entendiendo al cambio climático como un factor causal.

En este tema se aborda la estrategia de hospitales inteligentes, y según la Doctora Dana Van Alphen, asesora regional del Área de Preparativos para Situaciones de Emergencia y Socorro en casos de Desastre de la OPS/OMS, «el proyecto permitirá el desarrollo de un índice de instalaciones de salud inteligentes que acompañará a los estándares y códigos de construcción nacionales, además de una herramienta para apoyar en la implementación de medidas de mitigación frente al cambio climático en las instalaciones de salud existentes, así como en las que están por construirse». Por otro lado, este proyecto también propone entrenamientos y talleres de capacitación para mejorar las capacidades nacionales para lograr instalaciones de salud más preparadas frente al cambio climático, el fortalecimiento de las políticas en este ámbito, así como la asesoría del Grupo Asesor en Mitigación de Desastres de la OPS/ $\mathrm{OMS}^{10}$.

Esta iniciativa permitirá reducir los costos y las cuentas de los servicios públicos, limitar las emisiones de gases de efecto invernadero, mejorar la calidad del aire y la accesibilidad en los hospitales, así como el acceso a agua segura y las condiciones de seguridad. Por tanto, se consideró prioritario capacitar sobre este tópico a los profesionales encargados de las evaluaciones de los hospitales en México en el marco del Programa Hospitales Seguros.

Es cierto que la heterogeneidad de perfiles puede haber influido en que algunos contenidos les resultaran desconocidos o complejos. No obstante, es precisamente esta heterogeneidad una de las fortalezas del curso y del programa: el proceso mismo de evaluación incluye tanto temas administrativos como técnicos, clínicos y hasta relacionados directamente con las características geofísicas y el diseño estructural y la ingeniería de una instalación médica. Se pretende que las diversas disciplinas y áreas de conocimiento que estos participantes dominan se compaginen durante su participación, planeación para la respuesta a desastres y evaluación de la misma.

Es importante considerar que los alumnos que aprobaron el curso se consideran "candidatos a evaluador»; para llegar a ser evaluador es necesario que aprueben tres prácticas, una en cada rubro del Programa Hospital Seguro (Seguridad estructural, Seguridad no estructural, Capacidad funcional), con el fin tanto de reforzar los conocimientos como de verificar que pueden transpolarlos a la realidad. En este momento, los alumnos aprobados están realizando sus prácticas en todo el país, y los resultados serán motivo de otro estudio.

\section{Conflicto de intereses}

Los autores declaran no tener conflicto de intereses.

\section{Bibliografía}

1. Bitrán DB. El impacto de los desastres naturales en el desarrollo económico. 1992. (Consultado el 19 de diciembre de 2017.) Disponible en: http://www.eird.org/esp/cdcapra/pdf/spa/doc2194/doc2194-contenido.pdf.

2. Bello O, Ortiz L, Samaniego JL. La estimación del efecto de los desastres en América latina, 1972-2010. ONU-CEPAL; 2014.

3. Cicero Sabido R, Padua Gabriel A, Rodríguez Martínez H, Toledo B, Yáñez Villar A. Efectos del terremoto del 19 de septiembre de 1985 en el Hospital General de la Ciudad de México. Algunas consideraciones. Salud Pública de México. 1986;28:521-6. 
4. UNISDR. Terminología sobre reducción del riesgo de desastres. (Consultado el 16 de noviembre de 2017.) Disponible en: http://www.unisdr. org/files/7817_UNISDRTerminologySpanish.pdf.

5. Secretaría de Salud (México). La calidad de la atención a la salud en México a través de sus instituciones: 12 años de experiencia. México: Secretaría de Salud; 2012.

6. UNISDR. Marco de Sendai para la reducción del riesgo de desastres 2015-2030. (Consultado el 16 de noviembre de 2017.) Disponible en: https://www.unisdr.org/files/43291_spanishsendaiframeworkfordisasterri.pdf.

7. Cruz-Vega F, Loría-Castellanos J, Hernández-Olivas IP, Franco-Bey R, Ochoa-Ávila C, Sánchez-Badillo V. Experiencia en capacitación en emer- gencias de la División de Proyectos Especiales en Salud, Instituto Mexicano del Seguro Social. Cirugía y Cirujanos. 2016;84:127-34.

8. Loria-Castellanos J. Utilidad de una plataforma educativa en línea para la especialidad de urgencias médico-quirúrgicas en México. Rev Panam Salud Pública. 2014;35:374-9.

9. Moodle. Acerca de Moodle. (Consultado el 10 de noviembre de 2017.) Disponible en: https://docs.moodle.org/all/es/Acerca_de_Moodle.

10. El proyecto Hospitales Inteligentes mejorará la infraestructura sanitaria en el Caribe. (Consultado el 14 de diciembre de 2017.) Disponible en: http://www.paho.org/disasters/index.php?option=com_content\&view=article\&id=1700: smarthospitals\&ltemid=1178\&lang=es. 